\title{
Construction of Interval-valued Fuzzy Preference Relations from Ignorance Functions and Fuzzy Preference Relations. Application to Decision Making ${ }^{\text {th }}$
}

\author{
Edurne Barrenechea ${ }^{\mathrm{a}}$, Javier Fernandez ${ }^{\mathrm{a}}$, Miguel Pagola ${ }^{\mathrm{a}}$, Francisco Chiclana ${ }^{\mathrm{b}}$, \\ Humberto Bustince ${ }^{\mathrm{a}}$ \\ ${ }^{a}$ Research Group on Artificial Intelligence and Approximate Reasoning, Department of Automatic and \\ Computation, Public University of Navarra, Pamplona, Spain \\ ${ }^{b}$ Centre for Computational Intelligence (CCI) and DMU Interdisciplinary Group in Intelligent \\ Transport Systems (DIGITS), Faculty of Technology, De Montfort University, Leicester, UK
}

\begin{abstract}
This paper presents a method to construct an interval-valued fuzzy set from a fuzzy set and the representation of the lack of knowledge or ignorance that experts are subject to when they define the membership values of the elements to that fuzzy set. With this construction method, it is proved that membership intervals of equal length to the ignorance associated to the elements are obtained when the product t-norm and the probabilistic sum t-conorm are used. The construction method is applied to build interval-valued fuzzy preference relations $(\mathcal{I} \mathcal{V} \mathcal{P} \mathcal{R} s)$ from given fuzzy preference relations $(\mathcal{F} \mathcal{R} s)$. Afterwards, a general algorithm to solve decision making problems using $\mathcal{I V \mathcal { F } R} s$ is proposed. The decision making algorithm implements different selection processes of alternatives where the order used to choose alternatives is a key factor. For this reason, different admissible orders between intervals are analysed. Finally, OWA operators with interval weights are analysed and a method to obtain those weights from real-valued weights is proposed.
\end{abstract}

Keywords: Interval-valued fuzzy preference relation; Weak ignorance function; Admissible orders for intervals; Interval OWA operators; Interval weights; Decision making

\section{Introduction}

It is widely acknowledged that there exist problems for which the solution obtained using fuzzy techniques sometimes are very good, but some others not. Usually, this discordance is due to the experts' selection of a particular type of membership function to represent the information. For instance, in decision making problems, depending on the level of knowledge, experts might express their preferences using precise numerical values. Clearly, there are cases when the experts' level of knowledge about the environment where

\footnotetext{
${ }^{2}$ Cite as: Edurne Barrenechea, Javier Fernandez, Miguel Pagola, Francisco Chiclana, Humberto Bustince: Construction of Interval-valued Fuzzy Preference Relations from Ignorance Functions and Fuzzy Preference Relations. Knowledge-Based Systems. 58, March 2014, Pages 33-44. doi: 10.1016/j.knosys.2013.10.002

Email addresses: edurne.barrenechea@unavarra.es (Edurne Barrenechea), fcojavier.fernandez@unavarra.es ( Javier Fernandez), miguel.pagola@unavarra.es (Miguel Pagola), chiclana@dmu.ac.uk (Francisco Chiclana), bustince@unavarra.es (Humberto Bustince)
} 
the decision making is to be applied is rather low or imprecise. It may also happen, when alternatives are pairwise compared, that experts do not know with certainty whether they prefer one alternative to another, in which case they might choose to assign membership values close to 0.5 to reflect this ignorance situation. In these cases, it is appropriately argued though that precise numerical values to represent preferences might not be the most suitable choice in these cases, and hence the final decision arrived at might not the be the best one (see $[27,28,43])$.

Another difficulty arises sometimes in decision making: depending on the method used to solve a given problem it may happen that it is not possible to choose a single best alternative. For example, assume that we have the set of alternatives $X=\left\{x_{1}, x_{2}, x_{3}, x_{4}\right\}$ and the following fuzzy preference relation:

$$
\mathcal{R}=\left(\begin{array}{cccc}
- & 0.3149 & 0.1605 & 0.3640 \\
0.6851 & - & 0.0407 & 0.0624 \\
0.8395 & 0.9593 & - & 0.3874 \\
0.6360 & 0.9376 & 0.6126 & -
\end{array}\right)
$$

where $\mathcal{R}_{i j}$ represents the degree of preference of alternative $x_{i}$ over alternative $x_{j}$. If the well-known voting decision strategy that adds the preference values assigned to each alternative by rows and selects as best solution the alternative with the largest sum is applied, then both alternatives $x_{3}$ and $x_{4}$ are best in this particular scenario.

All previous considerations lead us to set the following research objectives:

1. To build, from fuzzy preference relations (fuzzy sets), interval-valued fuzzy preference relations (interval-valued fuzzy sets) where each element is associated an interval value representing the membership degree of that element to the set and with

(a) the original (numerical) membership value of the element to the set being a point of the interval value;

(b) and the length of the interval value represents the degree of uncertainty of the expert when giving the fuzzy membership degree (see [11, 42]).

2. To use different aggregation functions and methods for choosing the best alternative from an interval-valued fuzzy preference relation;

3. To analyse the influence of the order between intervals used for choosing the solution alternative.

Bivariate functions are proposed to achieve objective (1). The first value of the bivariate function will be the numerical value provided by the expert, i.e. the degree of membership of the element to the original $\mathcal{F} \mathcal{R}$; while the second value is derived from the ignorance function to represent the lack of knowledge of the expert in the assignation of the first value. From these two values, an $(\mathcal{I} \mathcal{V} \mathcal{F} \mathcal{R})$ is built. For objective (2), different approaches to select an alternative as solution are considered. In particular, interval-valued OWA operators with interval weights constructed from real weights and weak ignorance functions are proposed to generalise well-known non-dominance criteria. Finally, for objective (3), the processing of $\mathcal{I} \mathcal{V} \mathcal{F} \mathcal{R}$ makes necessary to deal with the issue of ordering interval values, for which admissible orders given by Bustince et al. in [12] are implemented in the decision making algorithm. 
This paper is organised as follows: To make the paper self-contained, in the next section we introduce the main concepts needed and used throughout it. In Section 3 we recall the concept of strict binary preference relation. Section 4 is devoted to the analysis of some properties of $\mathcal{I} \mathcal{V} \mathcal{F} \mathcal{R}$. Section 5 presents the construction method of interval values from two numerical values. This is used in Section 6 to obtain interval-valued fuzzy sets from fuzzy sets, whereas in Section 7 these results are used to build an $\mathcal{I} \mathcal{V} \mathcal{F} \mathcal{R}$ from an $\mathcal{F R}$. In Section 8 an algorithm for decision making that makes use of intervalvalued fuzzy sets is developed. In Section 9 consideration is given to the extension of the decision making algorithm to the following four well-known methods: the voting method, the method based on the use of real-valued OWA operators, the method based on the use of interval-valued OWA operators with interval-valued weights, and the non-dominance method. Section 10 proposes a consensus algorithm to apply in those cases when the outcomes of the different algorithms do not coincide. Section 11 provides an example to illustrate and make easier to understand the full extent of the application of all the developments of previous sections. Finally, conclusions are drawn in Section 12.

\section{Preliminary definitions}

In this section we recall some well-known definitions and results used throughout the paper.

A strictly decreasing and continuous function $\mathbf{N}:[0,1] \rightarrow[0,1]$ such that $\mathbf{N}(0)=1$ and $\mathbf{N}(1)=0$ is called a strict negation. If, in addition, it is involutive, i.e. $\mathbf{N}(\mathbf{N}(x))=x$ $\forall x \in[0,1]$, then $\mathbf{N}$ is said to be a strong negation. In this paper only strong negations will be considered.

Definition 1 (Fuzzy set). A fuzzy set $A$ on a finite universe $U \neq \emptyset$ is a mapping $A: U \rightarrow[0,1]$.

$W e$ denote by $\mathcal{F S}(U)$ the set of all fuzzy sets on $U$. Let us denote by $L([0,1])$ the set of all closed subintervals in $[0,1]$ :

$$
L([0,1])=\{\mathbf{x}=[\underline{x}, \bar{x}] \mid 0 \leq \underline{x} \leq \bar{x} \leq 1\} .
$$

Then $L([0,1])$ is a partially ordered set with respect to the relation $\leq_{L}$ defined in the following way: given $\mathbf{x}, \mathbf{y} \in L([0,1])$,

$$
\mathbf{x} \leq_{L} \mathbf{y} \text { if and only if } \underline{x} \leq \underline{y} \text { and } \bar{x} \leq \bar{y} .
$$

Moreover $\left(L([0,1]), \leq_{L}\right)$ is a complete lattice [19] with smallest element $0_{L}=[0,0]$ and largest element $1_{L}=[1,1]$. Note that it is not a linear lattice, since there are elements which are not comparable.

The following definition can be found in [9, 34] (see also [10, 20, 25, 51]).

Definition 2 (Interval-valued fuzzy set). An interval-valued fuzzy set (IVFS) (or interval type 2 fuzzy set) $A$ on a finite universe $U \neq \emptyset$ is a mapping $A: U \rightarrow L([0,1])$.

Let us denote by $W$ the function that associates to a closed subinterval of $[0,1]$ its length or width, i.e. $W: L([0,1]) \rightarrow[0,1]$ with $W([\underline{x}, \bar{x}])=\bar{x}-\underline{x}$. If $\mathbf{x}=[\underline{x}, \bar{x}] \in$ $L([0,1])$, by interval arithmetics [26] it is $1-\mathbf{x}=[1,1]-[\underline{x}, \bar{x}]=[1-\bar{x}, 1-\underline{x}]$ and we have that $W(\mathbf{x})=W(1-\mathbf{x})$. Note that given an IVFS A the membership of each element $u_{i} \in U$ is represented by an interval value $A\left(u_{i}\right)=\left[\underline{A}\left(u_{i}\right), \bar{A}\left(u_{i}\right)\right]$ with length 
$W\left(A\left(u_{i}\right)\right)=\bar{A}\left(u_{i}\right)-\underline{A}\left(u_{i}\right)$. A study on the evolution of the IVFSs and several results on their representation, connective types and operations can be found in $[9,19,35,45]$. We denote by $\mathcal{I} \mathcal{V} \mathcal{F} \mathcal{S}(U)$ the set of all IVFSs on $U$.

Definition 3 (Interval-valued negation). An interval-valued (IV) negation is a function $N_{I V}: L([0,1]) \rightarrow L([0,1])$ that is decreasing, with respect to $\leq_{L}$, with $N_{I V}\left(1_{L}\right)=0_{L}$ and $N_{I V}\left(0_{L}\right)=1_{L}$.

An IV negation $N_{I V}$ is said to be involutive if it verifies: $N_{I V}\left(N_{I V}(\mathbf{x})\right)=\mathbf{x}, \forall \mathbf{x} \in$ $L([0,1])$. The following result links involutive IV negations with strong negations:

Theorem 1 (Deschrijver, Cornelis and Kerre [19]). $N_{I V}$ is an involutive IV negation if and only if there exists a strong negation $\mathbf{N}$ such that $N_{I V}([\underline{x}, \bar{x}])=[\mathbf{N}(\bar{x}), \mathbf{N}(\underline{x})]$.

Throughout this paper we use the involutive IV negation $N_{I V}$ generated from the standard strong negation $\mathbf{N}(x)=1-x$, i.e. $N_{I V}([\underline{x}, \bar{x}])=[1-\bar{x}, 1-\underline{x}]$.

Definition 4 (Triangular norm). A triangular norm (t-norm) $T:[0,1]^{2} \rightarrow[0,1]$ is an associative, commutative, non-decreasing function such that $T(1, x)=x$ for all $x \in[0,1]$.

The three basic and mostly used t-norms are: the minimum $T_{M}(x, y)=\min (x, y)$, the product $T_{P}(x, y)=x \cdot y$ and the Eukasiewicz $T_{\mathrm{E}}(x, y)=\max (x+y-1,0)$. A t-norm $T$ is called idempotent if $T(x, x)=x$ for all $x \in[0,1]$. The only idempotent t-norm is the minimum. A t-norm $T$ is called strict if it is a strictly increasing function in each place on $] 0,1\left[^{2}\right.$. A t-norm $T$ is called nilpotent if it is continuous and each element $\left.a \in\right] 0,1[$ is a nilpotent element of $T$, i.e., if there exists an $n \in\{1,2, \ldots\}$ such that $T(\overbrace{a, \ldots, a}^{n-\text { times }})=0$ for any $a \in] 0,1[$.

Definition 5. An automorphism of the unit interval is a strictly increasing bijective mapping $\varphi:[0,1] \rightarrow[0,1]$.

Note that any automorphism $\varphi$ is continuous and: $\varphi(0)=0$ and $\varphi(1)=1$. We have the following representation theorem for nilpotent t-norms:

Theorem 2 (Klement, Mesiar and Pap [33]). For a function $T:[0,1]^{2} \rightarrow[0,1]$ the following statements are equivalent:

(i) $T$ is a nilpotent t-norm.

(ii) $T$ is isomorphic to the Eukasiewicz t-norm $T_{E}$, i.e., there is an automorphism $\varphi$ of the unit interval such that $\forall(x, y) \in[0,1]^{2}$

$$
T(x, y)=\varphi^{-1}\left(T_{E}(\varphi(x), \varphi(y))\right)=\varphi^{-1}(\max (\varphi(x)+\varphi(y)-1,0)) .
$$

In 1949, Aczél [1] proved the following theorem:

Theorem 3 (Aczél [1]). A continuous t-norm $T$ is strict if and only if there exists an automorphism $\varphi$ of the unit interval such that

$$
T(x, y)=\varphi^{-1}(\varphi(x) \cdot \varphi(y)) .
$$


Definition 6 (Triangular conorm). A triangular conorm (t-conorm) $S:[0,1]^{2} \rightarrow[0,1]$ is an associative, commutative, non-decreasing function such that $S(0, x)=x$ for all $x \in[0,1]$.

The three basic and mostly used t-conorms are: the maximum $S_{M}(x, y)=\max (x, y)$, the probabilistic sum $S_{+}(x, y)=x+y-x \cdot y$ and the Eukasiewicz $S_{\mathrm{E}}(x, y)=\min (x+y, 1)$. It is well known that t-norms and t-conorms on $[0,1]^{2}$ can be used to model the union and intersection, respectively, of fuzzy sets (see $[4,13,23]$ ). A t-norm $T$ and a t-conorm $S$ are dual with respect to the negation $\mathbf{N}$ if $T(x, y)=\mathbf{N}(S(\mathbf{N}(x), \mathbf{N}(y)))$ for all $x, y \in[0,1]$. For instance, $T_{P}$ and $S_{+}$are dual with respect to the standard strong negation.

In the following, we will extend these concepts to the case of interval-valued fuzzy sets $[9,19]$.

Definition 7. A function $\mathbf{T}: L([0,1])^{2} \rightarrow L([0,1])$ is said to be an interval-valued t-norm (IV t-norm) if it is commutative, associative, increasing in both arguments (with respect to the order $\left.\leq_{L}\right)$, and has the neutral element $1_{L}$. Similarly, a function $\mathbf{S}: L([0,1])^{2} \rightarrow$ $L([0,1])$ is said to be an interval-valued t-conorm (IV t-conorm) if it is commutative, associative, increasing, and has the neutral element $0_{L}$.

Definition 8. a) An $I V$ t-norm is said to be t-representable if there are two t-norms $T_{a}$ and $T_{b}$ in $[0,1]$ such that $\mathbf{T}(\mathbf{x}, \mathbf{y})=\left[T_{a}(\underline{x}, \underline{y}), T_{b}(\bar{x}, \bar{y})\right] \in L([0,1])$ for all $\mathbf{x}, \mathbf{y} \in$ $L([0,1])$.

b) An $I V$ t-conorm is said to be s-representable if there are two $t$-conorms $S_{a}$ and $S_{b}$ in $[0,1]$ such that $\mathbf{S}(\mathbf{x}, \mathbf{y})=\left[S_{a}(\underline{x}, \underline{y}), S_{b}(\bar{x}, \bar{y})\right] \in L([0,1])$ for all $\mathbf{x}, \mathbf{y} \in L([0,1])$.

In this paper we only use t-representable IV t-norms and s-representable IV t-conorms. In [12] the notion of admissible orders on $L([0,1])$ was introduced and investigated. The authors established that a binary relation $\preceq$ on $L([0,1])$ is an admissible order if it is a linear order on $L([0,1])$ refining $\leq_{L}$, i.e. if for all $[a, b],[c, d] \in L([0,1])$ such that $[a, b] \leq_{L}[c, d]$ then it is also $[a, b] \preceq[c, d]$. The use of admissible orders allow us to compare intervals using total orders between them. Next some examples of admissible orders are given.

Example 1. Let $[a, b],[c, d] \in L([0,1])$ :

- $[a, b] \preceq_{L 1}[c, d] \Leftrightarrow a<c$ or $(a=c$ and $b \leq d)$;

- $[a, b] \preceq_{L 2}[c, d] \Leftrightarrow b<d$ or $(b=d$ and $a \leq c)$;

- $[a, b] \preceq_{X Y}[c, d] \Leftrightarrow a+b<c+d$ or $(a+b=c+d$ and $b-a \leq d-c)$ (defined by $X u$ and Yager in [47]);

- $[a, b] \preceq_{\alpha, \beta}[c, d] \Leftrightarrow K_{\alpha}(a, b)<K_{\alpha}(c, d)$ or $\left(K_{\alpha}(a, b)=K_{\alpha}(c, d)\right.$ and $K_{\beta}(a, b) \leq$ $\left.K_{\beta}(c, d)\right)$, being $K_{\alpha}:[0,1]^{2} \rightarrow[0,1]$ a mapping defined by $K_{\alpha}(a, b)=a+\alpha \cdot(b-a)$ for $\alpha, \beta \in[0,1]$ and $\alpha \neq \beta$.

Definition 9. An n-ary $(n \in \mathbb{N}, n \geq 2)$ aggregation function is a non-decreasing mapping in each argument, $M:[0,1]^{n} \rightarrow[0,1]$, verifying $M(0, \cdots, 0)=0$ and $M(1, \cdots, 1)=1$.

Bustince et al. [12] described different construction methods of admissible orders by means of aggregation functions, as well as the notion of aggregation function of interval values. 
Definition 10 (Aggregation function of interval values). Let $(L([0,1]), \preceq)$ be a bounded partially ordered set (poset) with smallest element $0_{L}$ and greatest element $1_{L}$. A mapping $\mathcal{M}: L([0,1])^{n} \rightarrow L([0,1])$ is a n-ary $(n \in \mathbb{N}, n \geq 2)$ aggregation function on $(L([0,1]), \preceq)$ if it is $\preceq$-increasing, i.e., for all $\mathbf{x}, \mathbf{y} \in L([0,1])^{n}, \mathcal{M}(\mathbf{x}) \preceq \mathcal{M}(\mathbf{y})$ whenever $x_{1} \preceq y_{1}, \cdots, x_{n} \preceq y_{n}$, and satisfies the boundary conditions $\mathcal{M}\left(0_{L}, \cdots, 0_{L}\right)=0_{L}$, $\mathcal{M}\left(1_{L}, \cdots, 1_{L}\right)=1_{L}$.

The concept of ignorance functions is defined in [11] to measure the degree of ignorance/lack of knowledge of an expert when he/she assigns numerical value as membership degree of an object to a given class and another numerical value for the membership of the same element to a different class. An adaptation of this concept using a variable and its negation was defined as a weak ignorance function in [41].

Definition 11 (Sanz et al. [41] ). A weak ignorance function is a continuous mapping $g:[0,1] \rightarrow[0,1]$ verifying:

1. $g(x)=g(1-x)$ for all $x \in[0,1]$;

2. $g(x)=0$ if and only if $x=0$ or $x=1$;

3. $g(0.5)=1$.

Note that the symmetry property of function $g$ wrt to the value 0.5 makes the explicit declarations of properties to verify by $g$ be enough for one of the two subdomains $[0,0.5]$ or $[0.5,1]$. Consequently, if it is asserted that $g$ is a monotonic function then it is meant that $g$ is increasing (decreasing) in $[0,0.5]$ and decreasing (increasing) in $[0.5,1]$.

Example 2. The following function

$$
g(x)=2 \cdot \min (x, 1-x) \text { for all } x \in[0,1]
$$

is a weak ignorance function.

Given a fuzzy set $A \in \mathcal{F} \mathcal{S}(U)$ and a weak ignorance function $g$, the considerations and reasoning provided in [11] imply that the value $g\left(A\left(u_{i}\right)\right)$ represents the ignorance associate to the value $A\left(u_{i}\right)$.

\section{Strict Fuzzy binary preference relations}

A fuzzy preference relation $\mathcal{R}$ on a set of alternatives $X=\left\{x_{1}, \ldots, x_{n}\right\}$ is a fuzzy subset of the Cartesian product $X \times X$, that is $\mathcal{R}: X \times X \rightarrow[0,1]([14,16,23,27,37])$; for each pair of alternatives $x_{i}$ and $x_{j}, \mathcal{R}_{i j}=\mathcal{R}\left(x_{i}, x_{j}\right)$ represents a degree of (weak) preference of $x_{i}$ over $x_{j}$, namely the degree to which $x_{i}$ is considered as least as good as $x_{j}$ (by definition, $\mathcal{R}_{i i}=1$ ). The preference relation may be conveniently represented by the $n \times n$ matrix $\mathcal{R}=\left(\mathcal{R}_{i, j}\right)$ for all $i, j \in\{1, \ldots, n\}$. We denote by $\mathcal{F} \mathcal{R}(X \times X)$ the set of all the fuzzy preference relations on $X$.

From a weak preference relation $\mathcal{R}$, Fodor and Roubens [23] (see also [16]) derive the following relations:

1. Strict preference $\mathcal{P}_{i j}=\mathcal{P}\left(x_{i}, x_{j}\right)$ is a measure of strict preference of $x_{i}$ over $x_{j}$, indicating that $x_{i}$ is (weakly) preferred to $x_{j}$ but $x_{j}$ is not (weakly) preferred to $x_{i}$. 
2. Indifference $\mathcal{I}_{i j}=\mathcal{I}\left(x_{i}, x_{j}\right)$ is a measure of the simultaneous fulfillment of $\mathcal{R}_{i j}$ and $\mathcal{R}_{j i}$. Roughly speaking, $x_{i}$ and $x_{j}$ are considered equal in the sense that both $x_{i}$ is as good as $x_{j}$ and the other way around.

3. Incomparability $\mathcal{J}_{i j}=\mathcal{J}\left(x_{i}, x_{j}\right)$ is a measure of the incomparability of $x_{i}$ and $x_{j}$.

More specifically, Fodor and Roubens [23] propose the following expressions of the above relations in terms of a t-norm $T$ and a strict negation $\mathbf{N}$ :

$$
\begin{gathered}
\mathcal{P}_{i j}=T\left(\mathcal{R}_{i j}, \mathbf{N}\left(\mathcal{R}_{j i}\right)\right) \text { for all } i, j \in\{1, \ldots, n\} ; \\
\mathcal{I}_{i j}=T\left(\mathcal{R}_{i j}, \mathcal{R}_{j i}\right) \text { for all } i, j \in\{1, \ldots, n\} ; \\
\mathcal{J}_{i j}=T\left(\mathbf{N}\left(\mathcal{R}_{i j}\right), \mathbf{N}\left(\mathcal{R}_{j i}\right)\right) \text { for all } i, j \in\{1, \ldots, n\} .
\end{gathered}
$$

Fuzzy preference structures, $(\mathcal{P}, \mathcal{I}, \mathcal{J})$, have been studied deeply (see $[17,23,24,29,36$, $38,39])$.

We say that $\mathcal{R} \in \mathcal{F} \mathcal{R}(X \times X)$ satisfies the property of reciprocity if $\mathcal{R}_{i j}+\mathcal{R}_{j i}=1$ for all $i, j \in\{1, \cdots, n\}(i \neq j)$. In reciprocal preference relations it is usual practice not to define the elements of the main diagonal (see [31]).

Given a fuzzy preference relation $\mathcal{R}^{*} \in \mathcal{F} \mathcal{R}(X \times X)$, a reciprocal fuzzy preference relation $\mathcal{R}$ can be constructed using Eq. (5):

$$
\mathcal{R}_{i j}=\left\{\begin{array}{l}
\frac{\mathcal{R}_{i j}^{*}}{\mathcal{R}_{i j}^{*}+\mathcal{R}_{j i}^{*}} \text { if } \mathcal{R}_{i j}^{*}+\mathcal{R}_{j i}^{*} \neq 0 \\
0 \text { othercase }
\end{array}\right.
$$

In this paper we only work with reciprocal fuzzy preference relations. We have the following result:

Theorem 4. Let $\mathcal{R}$ be a reciprocal fuzzy preference relation and $\mathbf{N}(x)=1-x$ for all $x \in[0,1]$. Then,

$$
\mathcal{P}_{i j}=\mathcal{R}_{i j} \text { if and only if } T \equiv T_{M}
$$

for all $\mathcal{R}_{i j} \in \mathcal{R}$.

Proof. Reciprocity of $\mathcal{R}$ and definition of $\mathbf{N}$ implies

$$
\mathcal{P}_{i j}=T\left(\mathcal{R}_{i j}, \mathbf{N}\left(\mathcal{R}_{j i}\right)\right)=T\left(\mathcal{R}_{i j}, 1-\mathcal{R}_{j i}\right)=T\left(\mathcal{R}_{i j}, \mathcal{R}_{i j}\right) .
$$

Therefore we have the following equivalences:

$$
\mathcal{P}_{i j}=\mathcal{R}_{i j} \Leftrightarrow \mathcal{R}_{i j}=T\left(\mathcal{R}_{i j}, \mathcal{R}_{i j}\right) \Leftrightarrow T \text { is idempotent } \Leftrightarrow T \equiv T_{M}
$$

Orlovsky in [36] gives the following definition of strict fuzzy preference relation:

Definition 12 (Orlovsky [36]). Given a fuzzy preference relation $\mathcal{R} \in \mathcal{F} \mathcal{R}(X \times X)$ a strict fuzzy preference relation $\mathcal{R}^{s} \in \mathcal{F} \mathcal{R}(X \times X)$ is given by:

$$
\mathcal{R}_{i j}^{s}= \begin{cases}\mathcal{R}_{i j}-\mathcal{R}_{j i} & \text { if } \mathcal{R}_{i j}>\mathcal{R}_{j i} \\ 0 & \text { otherwise }\end{cases}
$$


Next, we present the relationship between the strict fuzzy preference relation (2) given by Fodor and Roubens and the one given by Orlovsky (6):

Theorem 5. Let $\mathcal{R}$ be a fuzzy preference relation and $\mathbf{N}(x)=1-x$ for all $x \in[0,1]$. The following equivalence holds:

$$
\mathcal{P}_{i j}=\mathcal{R}_{i j}^{s}, \forall i, j \in\{1, \ldots, n\} \Leftrightarrow T \equiv T_{E} .
$$

Proof. Firstly, the strict preference value $\mathcal{R}_{i j}^{s}$ can be written as follows:

$$
\mathcal{R}_{i j}^{s}=\max \left(\mathcal{R}_{i j}-\mathcal{R}_{j i}, 0\right)=\max \left(\mathcal{R}_{i j}+1-\mathcal{R}_{j i}-1,0\right)=T_{\mathrm{E}}\left(\mathcal{R}_{i j}, 1-\mathcal{R}_{j i}\right) .
$$

Secondly, because $\mathbf{N}(x)=1-x$ we have that $\mathcal{P}_{i j}=T\left(\mathcal{R}_{i j}, 1-\mathcal{R}_{j i}\right)$. Therefore, the following equivalence is true

$$
\mathcal{P}_{i j}=\mathcal{R}_{i j}^{s}, \forall i, j \in\{1, \ldots, n\} \Leftrightarrow T\left(\mathcal{R}_{i j}, 1-\mathcal{R}_{j i}\right)=T_{\mathrm{E}}\left(\mathcal{R}_{i j}, 1-\mathcal{R}_{j i}\right), \forall i, j \in\{1, \ldots, n\} .
$$

Consequently, we have proved that

$$
\mathcal{P}_{i j}=\mathcal{R}_{i j}^{s}, \forall i, j \in\{1, \ldots, n\} \Leftrightarrow T \equiv T_{€}
$$

It is worth remarking that the fuzzy preference relation, $\mathcal{R}$, in Theorem 5 does not necessarily need to be reciprocal.

\section{Interval-valued fuzzy binary preference relations. Interval-valued strict preference, interval-valued indifference and interval-valued incomparability}

An approach that adds flexibility to represent uncertainty in decision making problems consists of using interval-valued fuzzy relations $[25,44,46]$. An interval-valued fuzzy preference relation $\widetilde{\mathcal{R}}$ on $X$ is defined as an interval-valued fuzzy subset of $X \times X$; that is, $\widetilde{\mathcal{R}}: X \times X \rightarrow L([0,1])$. The interval $\widetilde{\mathcal{R}}\left(x_{i}, x_{j}\right)=\mathbf{r}_{i j}=\left[\underline{r}_{i j}, \bar{r}_{i j}\right]$ denotes the degree to which elements $x_{i}$ and $x_{j}$ are related (representing the degree of preference of $x_{i}$ over $x_{j}$ ) in the relation $\widetilde{\mathcal{R}}$ for all $x_{i}, x_{j} \in X$. As usual, the elements of the main diagonal of an interval-baled fuzzy preference relation will not be considered and therefore in the rest of the paper we are assuming that $i \neq j$.

By $\mathcal{I} \mathcal{F} \mathcal{F}(X \times X)$ we denote the set of all interval-valued fuzzy preference relations on $X \times X$.

Definition 13. Let $\widetilde{\mathcal{R}} \in \mathcal{I} \mathcal{V F \mathcal { R }}(X \times X)$. We say that $\widetilde{\mathcal{R}}$ satisfies the reciprocity property if for all $\mathbf{r}_{i j}, \mathbf{r}_{j i} \in \widetilde{\mathcal{R}}$ the following identities hold:

$$
\begin{aligned}
& \underline{r}_{i j}+\bar{r}_{j i}=1 \\
& \underline{r}_{j i}+\bar{r}_{i j}=1
\end{aligned}
$$

For a comprehensive study on interval-valued fuzzy relations the reader is advised to read the following literature $[5-7,18]$.

Given an IVFR, $\widetilde{\mathcal{R}}=\left(\mathbf{r}_{i j}\right)$, in the following we define its corresponding Interval-valued strict preference $(\mathbb{P})$, interval-valued indifference $(\mathbb{I})$ and interval-valued incomparability $(\mathbb{J})$. These concepts are based on the corresponding ones given by Fodor and Roubens : strict preference $\mathcal{P}$ (eq. (2)), indifference $\mathcal{I}$ (eq. (3)), and incomparability $\mathcal{J}$ (eq. (4)), respectively. The new concepts here presented are based on the previously given concepts of t-representable IV t-norms (Definition 8) and IV negations (Theorem 1) generated from the standard strict negation: 
(1) Interval-valued strict preference

$$
\begin{aligned}
\mathbb{P}_{i j} & =T_{I V}\left(\mathbf{r}_{i j}, N_{I V}\left(\mathbf{r}_{j i}\right)\right)= \\
& =T_{I V}\left(\left[\underline{r}_{i j}, \bar{r}_{i j}\right],\left[1-\bar{r}_{j i}, 1-\underline{r}_{j i}\right]\right)= \\
& =\left[T_{a}\left(\underline{r}_{i j}, 1-\bar{r}_{j i}\right), T_{b}\left(\bar{r}_{i j}, 1-\underline{r}_{j i}\right)\right] \text { for all } i, j \in\{1, \ldots, n\},
\end{aligned}
$$

(2) Interval-valued indifference

$$
\begin{aligned}
\mathbb{I}_{i j} & =T_{I V}\left(\mathbf{r}_{i j}, \mathbf{r}_{j i}\right)= \\
& =T_{I V}\left(\left[\underline{r}_{i j}, \bar{r}_{i j}\right],\left[\underline{r}_{j i}, \bar{r}_{j i}\right]\right)= \\
& =\left[T_{a}\left(\underline{r}_{i j}, \underline{r}_{j i}\right), T_{b}\left(\bar{r}_{i j}, \bar{r}_{j i}\right)\right] \text { for all } i, j \in\{1, \ldots, n\},
\end{aligned}
$$

(3) Interval-valued incomparability

$$
\begin{aligned}
\mathbb{J}_{i j} & =T_{I V}\left(N_{I V}\left(\mathbf{r}_{i j}\right), N_{I V}\left(\mathbf{r}_{j i}\right)\right)= \\
& =T_{I V}\left(N_{I V}\left(\left[\underline{r}_{i j}, \bar{r}_{i j}\right]\right), N_{I V}\left(\left[\underline{r}_{j i}, \bar{r}_{j i}\right]\right)\right)= \\
& =\left[T_{a}\left(1-\bar{r}_{i j}, 1-\bar{r}_{j i}\right), T_{b}\left(1-\underline{r}_{i j}, 1-\underline{r}_{j i}\right)\right] \text { for all } i, j \in\{1, \ldots, n\} .
\end{aligned}
$$

It is necessary to notice that these concepts can be generalised for any IV t-norms and IV negation. The following results extends Theorem 4 to the case of IVFRs.

Theorem 6. Let $\widetilde{\mathcal{R}} \in \mathcal{I} \mathcal{V F} \mathcal{R}(X \times X)$ be reciprocal and let $\mathbb{P}_{i j}$ be its associated intervalvalued strict fuzzy preference relation given by Eq. (8). The following equivalence holds:

$$
\mathbb{P}_{i j}=\mathbf{r}_{i j} \text { for all } i, j \in\{1, \ldots, n\} \Leftrightarrow T_{a}=T_{b}=T_{M} .
$$

Proof. Fistly, note that because $\widetilde{\mathcal{R}}$ is reciprocal we have:

$$
\mathbb{P}_{i j}=\left[T_{a}\left(\underline{r}_{i j}, 1-\bar{r}_{j i}\right), T_{b}\left(\bar{r}_{i j}, 1-\underline{r}_{j i}\right)\right]=\left[T_{a}\left(\underline{r}_{i j}, \underline{r}_{i j}\right), T_{b}\left(\bar{r}_{i j}, \bar{r}_{i j}\right)\right]
$$

Secondly,

$$
\mathbb{P}_{i j}=\mathbf{r}_{i j} \text { for all } i, j \in\{1, \ldots, n\}
$$

is equivalent to

$$
\forall i, j: T_{a}\left(\underline{r}_{i j}, \underline{r}_{i j}\right)=\underline{r}_{i j} \wedge T_{b}\left(\bar{r}_{i j}, \bar{r}_{i j}\right)=\bar{r}_{i j}
$$

Thus, both $T_{a}$ and $T_{b}$ are idempotent t-norms and consequently, because there is only one idempotent t-norm, both are the same and equal to the minimum t-norm:

$$
T_{a}=T_{b}=T_{M}
$$

\section{Construction of intervals from two numbers}

Our aim in this section is to build elements of $L([0,1])$, satisfying a determined set of properties, by means of two numbers in $[0,1]$ such that: (i) The first value is a point inside the interval; (ii) The second value is proportional to the width of the interval.

For this reason we consider functions of the type:

$$
\begin{aligned}
F:[0,1]^{2} & \rightarrow L([0,1]) \\
F(x, y) & =[\underline{F}(x, y), \bar{F}(x, y)]
\end{aligned}
$$

verifying 
(A) $x \in F(x, y)$ for all $x, y \in[0,1]$;

(B) If $y_{1} \leq y_{2}$, then $W\left(F\left(x, y_{1}\right)\right) \leq W\left(F\left(x, y_{2}\right)\right)$ for all $x, y_{1}, y_{2} \in[0,1]$;

The following two properties are also demanded:

(C) $F(x, 0)=[x, x]$ and $F(x, 1)=[0,1]$ for all $x \in[0,1]$;

(D) For all $x, y \in[0,1]$ we have

$$
\begin{aligned}
& \underline{F}(x, y)+\bar{F}(1-x, y)=1 \\
& \bar{F}(x, y)+\underline{F}(1-x, y)=1 .
\end{aligned}
$$

Note that property (D) is related with the reciprocity property of preferences. An example of such a function $F$ is provided next.

Example 3. The following function satisfies properties (A)-(D):

$$
F(x, y)=\left\{\begin{array}{ll}
{[x, x]} & \text { if } y \neq 1 \\
{[0,1]} & \text { if } y=1
\end{array} .\right.
$$

I n the following we will study function $F$ that are constructed using aggregation functions for both $\underline{F}$ and $\bar{F}$ functions. This is later followed by a characterisation of functions $F$ using t-norms and t-conorms.

Let $M_{1}$ and $M_{2}$ be two binary aggregation functions with function $F$ defined as follows:

$$
F_{M_{1}, M_{2}}(x, y)=\left[M_{1}(x, y), M_{2}(x, y)\right] .
$$

We observe that function $F$ constructed as above does not satisfy property (C). Indeed, if property (C) was true then it would also be true that

$$
M_{1}(x, 1)=0 \forall x \in[0,1] .
$$

In particular, we would have that $M_{1}(1,1)=0$, in contradiction with the fact that $M_{1}(1,1)=1$.

We note that if $M_{1}(x, 1-y)$ is used as the first argument of function $F$ instead of $M_{1}(x, y)$ then we would avoid the particular contradiction of $M_{1}(1,1)=0$ found above, although complementary conditions are necessary to fulfil properties (A)-(D). This is expressed in the following result.

Proposition 1. Let $M_{1}$ and $M_{2}$ be two aggregation functions such that $M_{1}(x, 1-y) \leq$ $M_{2}(x, y)$ for all $x, y \in[0,1]$. Consider the function

$$
\begin{aligned}
& F_{M_{1}, M_{2}}:[0,1]^{2} \rightarrow L([0,1]) \\
& F_{M_{1}, M_{2}}(x, y)=\left[M_{1}(x, 1-y), M_{2}(x, y)\right] .
\end{aligned}
$$

Then it holds that:

1. $F_{M_{1}, M_{2}}$ satisfies $(A)$ if and only if $M_{1}(x, 1) \leq x$ and $M_{2}(x, 0) \geq x$ for all $x \in[0,1]$;

2. $F_{M_{1}, M_{2}}$ satisfies $(B)$; 
3. $F_{M_{1}, M_{2}}$ satisfies $(C)$ if and only if $M_{1}(x, 1)=x, M_{1}(x, 0)=0, M_{2}(x, 0)=x$ and $M_{2}(x, 1)=1$ for all $x \in[0,1]$;

4. $F_{M_{1}, M_{2}}$ satisfies (D) if and only if $M_{1}$ and $M_{2}$ are dual with respect to the standard negation.

Proof. Direct.

The following result states that function $F$ can be constructed using a pair of dual t-norm and t-conorm.

Proposition 2. Let $(T, S)$ a pair of dual t-norm and a t-conorm with respect to the standard strong negation, $\mathbf{N}(x)=1-x$. The function

$$
\begin{aligned}
& F_{T, S}:[0,1]^{2} \rightarrow L([0,1]) \\
& F_{T, S}(x, y)=[T(x, 1-y), S(x, y)]
\end{aligned}
$$

satisfies properties $(A),(B),(C),(D)$ and $F_{T, S}(0, y)=[0, y]$ for all $y \in[0,1]$.

Proof. Note that $T$ is a t-norm and therefore we have that $0 \leq T(x, 1-y) \leq \min (x, 1-y)$. Also, $S$ is a t-conorm and therefore $\max (x, y) \leq S(x, y) \leq 1$. The following inequality $\min (x, 1-y) \leq x \leq \max (x, y)$ implies that $F_{T, S}(x, y) \in L([0,1])$, and therefore $F$ is well defined. It also implies that $x \in F_{T, S}(x, y)$, and therefore property (A) is proved. The proof of properties (B), (C) and (D) are direct and left for the reader.

The following results characterises the t-norms and t-conorms to use in function $F_{T, S}$ to produce intervals of length equal to the second argument $y$.

Theorem 7. Let $(T, S)$ a pair of dual t-norm and a t-conorm with respect to the standard strong negation, $\mathbf{N}(x)=1-x$, with $T$ being continuous and strict. Then:

$$
y=W\left(F_{T, S}(x, y)\right) \forall y \in[0,1] \Leftrightarrow T \equiv T_{P} \wedge S \equiv S_{+}
$$

Proof. Starting with $y=W\left(F_{T, S}(x, y)\right)$, and using the duality property between $T$ and $S$ we have:

$y=S(x, y)-T(x, 1-y)=1-T(1-x, 1-y)-T(x, 1-y) \Leftrightarrow T(x, 1-y)+T(1-x, 1-y)=1-y$.

Denoting $a=1-y, b=x$ and taking into account the symmetry of $T$, we have that

$$
T(a, b)+T(a, 1-b)=a \text { for all } a, b \in[0,1]
$$

Because $T$ is strict and continuous, by Theorem 3, there is an automorphism $\varphi$ of the unit interval such that

$$
T(a, b)=\varphi^{-1}(\varphi(a) \cdot \varphi(b)) \forall a, b \in[0,1]
$$

From [23], Eq. (12) holds if and only if $\varphi(x)=x^{p}$ for some $\left.p \in\right] 0, \infty[$, and then $T(a, b)=$ $\varphi^{-1}(\varphi(a) \cdot \varphi(b))=\left(a^{p} \cdot b^{p}\right)^{\frac{1}{p}}=a \cdot b$. Consequently, we have that $T \equiv T_{P}$, which completes the proof. 
Corollary 1. In the setting of Theorem 7 it is true that

$$
y=W\left(F_{T, S}(x, y)\right)=W\left(F_{T, S}(\mathbf{N}(x), y)\right) \forall x, y \in[0,1]
$$

with $\mathbf{N}$ any negation operator.

Proof. Note that this result is obvious because the length of the interval constructed with the function $F_{T, S}$ is equal to $y$ and this value is not affected by the $x$ value when $y$ is fixed.

The main contribution of the method contained in Theorem 7 is that an intervalvalued fuzzy set can be constructed from a fuzzy set. Indeed, given $A \in \mathcal{F} \mathcal{S}(U)$ an interval-valued fuzzy set $\widetilde{A} \in \mathcal{I} \mathcal{V} \mathcal{F} \mathcal{S}(U)$ can be obtained using an ignorance function $g$, by assigning to each element $u_{i} \in U$ the following membership interval value: $\left[T_{P}\left(A\left(u_{i}\right), 1-\right.\right.$ $\left.\left.g\left(A\left(u_{i}\right)\right)\right), S_{+}\left(A\left(u_{i}\right), g\left(A\left(u_{i}\right)\right)\right)\right]$.

\section{Construction of fuzzy sets from interval-valued fuzzy sets}

In 1983 Atanassov introduced a new operator [2, 3] allowing to associate a fuzzy set with each Atanassov intuitionistic fuzzy set or interval-valued fuzzy set. In fact, this operator takes a value from the interval representing the membership to the IVFS and defines that value to be the membership degree to a fuzzy set.

Definition 14. The operator $K$ colon $L([0,1]) \rightarrow[0,1]$ is given by $K=\left(K_{\alpha}\right)_{\alpha \in[0,1]}$, with each operator $K_{\alpha}: L([0,1]) \rightarrow[0,1]$ defined as a convex combination of its boundary arguments by $K_{\alpha}(\mathbf{x})=\underline{x}+\alpha(\bar{x}-\underline{x})$.

A different and new operator $P: L([0,1]) \rightarrow[0,1]$ to associate a fuzzy set with each interval-valued fuzzy set is proposed here:

$$
P(\mathbf{x})= \begin{cases}\frac{\underline{x}}{1-W(\mathbf{x})} & \text { if } W(\mathbf{x}) \neq 1 \\ 0 & \text { if } W(\mathbf{x})=1\end{cases}
$$

Recall that for $\mathbf{x}=[\underline{x}, \bar{x}]$, we have that $W(\mathbf{x})=\bar{x}-\underline{x}$ is the width or length of $\mathbf{x}$.

The following set of properties tare verified by $P$ :

Proposition 3. Operator $P$ verifies the following:

1. $\underline{x} \leq P(\mathbf{x}) \leq \bar{x}$ for all $\mathbf{x} \in L([0,1])$;

2. $P([x, x])=x$ for all $x \in[0,1]$;

3. If $W(\mathbf{x}) \neq 1$, then $P(\mathbf{x})+P(1-\mathbf{x})=1$;

4. If $W(\mathbf{x})=1$, then $P(\mathbf{x})+P(1-\mathbf{x})=0$.

Proof. 1. From Eq. (14) we have $\underline{x}=P(\mathbf{x})(1-W(\mathbf{x})) \leq P(\mathbf{x})$. Besides $\bar{x}=\underline{x}+$ $W(\mathbf{x})=P(\mathbf{x})(1-W(\mathbf{x}))+W(\mathbf{x})=P(\mathbf{x})+W(\mathbf{x})(1-P(\mathbf{x})) \geq P(\mathbf{x})$. Obviously if $W(\mathbf{x})=1$, then $\mathbf{x}=[0,1]$ and therefore $P(\mathbf{x}) \in[0,1]$.

2. If we take $\mathbf{x}=[x, x]$, then $W(\mathbf{x})=0$, therefore by Eq. (14) we have $P([x, x])=x$. 
3. We know that $W(\mathbf{x})=W(1-\mathbf{x})$, therefore $P(\mathbf{x})+P(1-\mathbf{x})=\frac{\underline{x}+1-\bar{x}}{1-W(\mathbf{X})}=\frac{1-(\bar{x}-\underline{x})}{1-W(\mathbf{X})}=1$.

4. If $W(\mathbf{x})=1$, then by Eq. (14), we obtain directly that $P(\mathbf{x})+P(1-\mathbf{x})=0$.

Corollary 2. In the setting of Theorem 7 , the following properties hold:

1. $P\left(F_{T_{P}, S_{+}}(x, y)\right)=x$ for all $x \in[0,1]$ and for all $y \in[0,1)$;

2. $P\left(F_{T_{P}, S_{+}}(x, 1)\right)=0$ for all $x \in[0,1]$.

Proof. $\quad$ 1. $P\left(F_{T_{P}, S_{+}}(x, y)\right)=P([x(1-y), x(1-y)+y])=\frac{x(1-y)}{(1-y)}=x$ for all $x \in[0,1]$ and $y \in[0,1)$.

2. If $y=1$, then from Eq. (14) we have $P\left(F_{T_{P}, S_{+}}(x, 1)\right)=P([0,1])=0$.

The following results provides a method to derive a fuzzy sets $A \mathcal{F} \mathcal{S}(U)$ from an interval-valued fuzzy set $\widetilde{A} \in \mathcal{I} \mathcal{V} \mathcal{F} \mathcal{S}(U)$.

Proposition 4. Given an interval-valued fuzzy set $\widetilde{A} \in \mathcal{I V F} \mathcal{S}(U)$, the following

$$
A=\left\{\left(u_{i}, P\left(\widetilde{A}\left(u_{i}\right)\right)\right) \mid u_{i} \in U\right\}
$$

is a fuzzy set on $U$.

Proof. Direct.

\section{Construction of interval-valued fuzzy preference relations from fuzzy rela- tions and weak ignorance functions}

The aim of this section is to build an interval-valued fuzzy preference relation $\widetilde{\mathcal{R}}=\left(\mathbf{r}_{i j}\right)$ from a given fuzzy preference relation $\mathcal{R}=\left(\mathcal{R}_{i j}\right)$. For this purpose, the construction method of intervals presented in Section 5 will be used with function $F_{T, S}$ and following argument values:

- $\mathcal{R}_{i j}$ - the value given by an expert representing the degree of preference of alternative $x_{i}$ over alternative $x_{j}$; and

- $g\left(\mathcal{R}_{i j}\right)$ - the lack of knowledge (ignorance) of the expert when he/she provides $\mathcal{R}_{i j}$.

Moreover, $\widetilde{\mathcal{R}}$ will have elements with length equal to the weak ignorance associated with the membership degree of the same element of the original fuzzy preference relation $\mathcal{R}$.

Proposition 5. Let $\widetilde{\mathcal{R}}=\left(\mathbf{r}_{i j}\right)$ be defined as follows

$$
\mathbf{r}_{i j}=F_{T, S}\left(\mathcal{R}_{i j}, g\left(\mathcal{R}_{i j}\right)\right) \forall i, j \in\{1, \cdots, n\},
$$

with $\mathcal{R} \in \mathcal{F} \mathcal{R}(X \times X)$ being a fuzzy preference relation on $X \times X$, and $g$ be a weak ignorance function. The following statements hold:

1. $\widetilde{\mathcal{R}}$ is an interval-valued fuzzy preference relation on $X \times X$; 
2. $W\left(\mathbf{r}_{i j}\right)=W\left(F_{T_{P}, S_{+}}\left(\mathcal{R}_{i j}, g\left(\mathcal{R}_{i j}\right)\right)\right)=g\left(\mathcal{R}_{i j}\right)$ for all $\mathcal{R}_{i j} \in \mathcal{R}$;

3. $P\left(\mathbf{r}_{i j}\right)=P\left(F_{T_{P}, S_{+}}\left(\mathcal{R}_{i j}, g\left(\mathcal{R}_{i j}\right)\right)\right)=\mathcal{R}_{i j}$ for all $g\left(\mathcal{R}_{i j}\right) \neq 1$.

4. If $g\left(\mathcal{R}_{i j}\right)=1$ then, $P\left(\mathbf{r}_{i j}\right)=P\left(F_{T_{P}, S_{+}}\left(\mathcal{R}_{i j}, g\left(\mathcal{R}_{i j}\right)\right)\right)=0$.

Proof. This is left for the reader as it is derivative from Proposition 2, Theorem 7, and Corollary 2.

The following result guarantees that $\widetilde{\mathcal{R}} \in \mathcal{I} \mathcal{V} \mathcal{F} \mathcal{R}(X \times X)$ is reciprocal when $\mathcal{R} \in$ $\mathcal{F R}(X \times X)$ is reciprocal.

Proposition 6. Let $g$ be a weak ignorance function and $\mathcal{R} \in \mathcal{F} \mathcal{R}(X \times X)$. If $\mathcal{R}$ is reciprocal then $\widetilde{\mathcal{R}} \in \mathcal{I} \mathcal{V} \mathcal{F} \mathcal{R}(X \times X)$ with elements

$$
\mathbf{r}_{i j}=F_{T_{P}, S_{+}}\left(\mathcal{R}_{i j}, g\left(\mathcal{R}_{i j}\right)\right) \forall i, j \in\{1, \cdots, n\}
$$

is reciprocal.

Proof. For being $g$ a weak ignorance function it is (see Definition 11):

$$
g\left(\mathcal{R}_{i j}\right)=g\left(1-\mathcal{R}_{i j}\right) \forall i, j \in\{1, \cdots, n\} .
$$

Reciprocity of $\mathcal{R}$ implies

$$
g\left(\mathcal{R}_{i j}\right)=g\left(\mathcal{R}_{j i}\right)
$$

By definition we have:

$$
\mathbf{r}_{i j}=\left[\mathcal{R}_{i j} \cdot\left(1-g\left(\mathcal{R}_{i j}\right)\right), \mathcal{R}_{i j} \cdot\left(1-g\left(\mathcal{R}_{i j}\right)\right)+g\left(\mathcal{R}_{i j}\right)\right]
$$

and

$$
\mathbf{r}_{j i}=\left[\mathcal{R}_{j i} \cdot\left(1-g\left(\mathcal{R}_{j i}\right)\right), \mathcal{R}_{j i} \cdot\left(1-g\left(\mathcal{R}_{j i}\right)\right)+g\left(\mathcal{R}_{j i}\right)\right]
$$

Hence:

$$
\begin{aligned}
\underline{r}_{i j}+\bar{r}_{j i} & =\mathcal{R}_{i j} \cdot\left(1-g\left(\mathcal{R}_{i j}\right)\right)+\mathcal{R}_{j i} \cdot\left(1-g\left(\mathcal{R}_{j i}\right)\right)+g\left(\mathcal{R}_{j i}\right) \\
& =\mathcal{R}_{i j} \cdot\left(1-g\left(\mathcal{R}_{i j}\right)\right)+\mathcal{R}_{j i} \cdot\left(1-g\left(\mathcal{R}_{i j}\right)\right)+g\left(\mathcal{R}_{i j}\right) \\
& =\left(1-g\left(\mathcal{R}_{i j}\right)\right) \cdot\left(\mathcal{R}_{i j}+\mathcal{R}_{j i}\right)+g\left(\mathcal{R}_{i j}\right) \\
& =1-g\left(\mathcal{R}_{i j}\right)+g\left(\mathcal{R}_{i j}\right)=1
\end{aligned}
$$

Similarly, we prove that $\bar{r}_{i j}+\underline{r}_{j i}=1$.

The following result provides conditions for a weak ignorance function to guarantee that a fuzzy preference relations $\mathcal{R} \in \mathcal{F} \mathcal{R}(X \times X)$ is reciprocal.

Proposition 7. Let $\mathcal{R} \in \mathcal{F} \mathcal{R}(X \times X)$ be a fuzzy preference relation with $t R_{i j} \neq$ 0.5 , i.e. $R_{i j} \neq R_{j i}, \forall i, j$. If a weak ignorance function $g$ is monotonic and verifies that $g\left(\mathcal{R}_{i j}\right)=g\left(\mathcal{R}_{j i}\right)(\forall i, j)$, then $\mathcal{R}$ is reciprocal. 
Proof. Without loss of generality we can assume that $\mathcal{R}_{i j}>0.5$. From Definition 11, item 1., it is

$$
g\left(\mathcal{R}_{i j}\right)=g\left(1-\mathcal{R}_{i j}\right) .
$$

As we are assuming that $g\left(\mathcal{R}_{i j}\right)=g\left(\mathcal{R}_{j i}\right)(\forall i, j)$, then it is

$$
g\left(\mathcal{R}_{j i}\right)=g\left(1-\mathcal{R}_{i j}\right) .
$$

Note that the restriction of $g$ in $[0,0.5]$ is bijective because it is continuous and monotonic. Therefore, it is

$$
\mathcal{R}_{j i}=1-\mathcal{R}_{i j}
$$

The following result is a consequence of Propositions 6 and 7.

Corollary 3. Let $\mathcal{R} \in \mathcal{F} \mathcal{R}(X \times X)$ be a fuzzy preference relation with $R_{i j} \neq 0.5$, i.e. $R_{i j} \neq$ $R_{j i}, \forall i, j$. If a weak ignorance function $g$ is monotonic, then we have that

$$
g\left(\mathcal{R}_{i j}\right)=g\left(\mathcal{R}_{j i}\right) \forall i, j \Leftrightarrow \widetilde{\mathcal{R}}=\left(\mathbf{r}_{i j}\right) \in \mathcal{I} \mathcal{V} \mathcal{F} \mathcal{R}(X \times X) \text { is reciprocal }
$$

with $\mathbf{r}_{i j}=F_{T_{P}, S_{+}}\left(\mathcal{R}_{i j}, g\left(\mathcal{R}_{i j}\right)\right) \forall i, j$.

Example 4. Let $X=\left\{x_{1}, x_{2}, x_{3}, x_{4}\right\}$ be the set of alternatives. Consider the following reciprocal fuzzy preference relation $\mathcal{R}$ :

$$
\mathcal{R}=\left(\begin{array}{cccc}
- & 0.3149 & 0.1605 & 0.3640 \\
0.6851 & - & 0.0407 & 0.0624 \\
0.8395 & 0.9593 & - & 0.3874 \\
0.6360 & 0.9376 & 0.6126 & -
\end{array}\right)
$$

Take as weak ignorance function: $g(x)=2 \cdot \min (x, 1-x)$. The reciprocal interval-valued fuzzy relation $\widetilde{\mathcal{R}}$ generated by $F_{T_{P}, S_{+}}\left(\mathcal{R}_{i j}, g\left(\mathcal{R}_{i j}\right)\right)$ is:

$$
\begin{aligned}
& \mathbf{r}_{12}=F_{T_{P}, S_{+}}\left(\mathcal{R}_{12}, g\left(\mathcal{R}_{12}\right)\right)=F_{T_{P}, S_{+}}(0.3149,0.6298)=[0.1166,0.7464] ; \\
& \mathbf{r}_{13}=F_{T_{P}, S_{+}}\left(\mathcal{R}_{13}, g\left(\mathcal{R}_{13}\right)\right)=F_{T_{P}, S_{+}}(0.1605,0.3210)=[0.1090,0.4300] ; \\
& \mathbf{r}_{14}=F_{T_{P}, S_{+}}\left(\mathcal{R}_{14}, g\left(\mathcal{R}_{14}\right)\right)=F_{T_{P}, S_{+}}(0.3640,0.7280)=[0.0990,0.8270] ; \\
& \mathbf{r}_{21}=F_{T_{P}, S_{+}}\left(\mathcal{R}_{21}, g\left(\mathcal{R}_{21}\right)\right)=F_{T_{P}, S_{+}}(0.6851,0.6298)=[0.2536,0.8834] ; \\
& \mathbf{r}_{23}=F_{T_{P}, S_{+}}\left(\mathcal{R}_{23}, g\left(\mathcal{R}_{23}\right)\right)=F_{T_{P}, S_{+}}(0.0407,0.0814)=[0.0374,0.1188] ; \\
& \mathbf{r}_{24}=F_{T_{P}, S_{+}}\left(\mathcal{R}_{24}, g\left(\mathcal{R}_{24}\right)\right)=F_{T_{P}, S_{+}}(0.0624,0.1248)=[0.0546,0.1794] ; \\
& \mathbf{r}_{31}=F_{T_{P}, S_{+}}\left(\mathcal{R}_{31}, g\left(\mathcal{R}_{31}\right)\right)=F_{T_{P}, S_{+}}(0.8395,0.3210)=[0.5700,0.8910] ; \\
& \mathbf{r}_{32}=F_{T_{P}, S_{+}}\left(\mathcal{R}_{32}, g\left(\mathcal{R}_{32}\right)\right)=F_{T_{P}, S_{+}}(0.9593,0.0814)=[0.8812,0.9626] ; \\
& \mathbf{r}_{34}=F_{T_{P}, S_{+}}\left(\mathcal{R}_{34}, g\left(\mathcal{R}_{34}\right)\right)=F_{T_{P}, S_{+}}(0.3874,0.7748)=[0.0872,0.8620] ; \\
& \mathbf{r}_{41}=F_{T_{P}, S_{+}}\left(\mathcal{R}_{41}, g\left(\mathcal{R}_{41}\right)\right)=F_{T_{P}, S_{+}}(0.6360,0.7280)=[0.1730,0.9010] ; \\
& \mathbf{r}_{43}=F_{T_{P}, S_{+}}\left(\mathcal{R}_{42}, g\left(\mathcal{R}_{42}\right)\right)=F_{T_{P}, S_{+}}\left(\mathcal{R}_{43}, g\left(\mathcal{R}_{43}\right)\right)=F_{T_{P}, S_{+}}(0.9376,0.1248)=[0.8206,0.9454] ;
\end{aligned}
$$

$$
\widetilde{\mathcal{R}}=\left(\begin{array}{cccc}
- & {[0.1166,0.7464]} & {[0.1090,0.4300]} & {[0.0990,0.8270]} \\
{[0.2536,0.8834]} & - & {[0.0374,0.1188]} & {[0.0546,0.1794]} \\
{[0.5700,0.8910]} & {[0.8812,0.9626]} & - & {[0.0872,0.8620]} \\
{[0.1730,0.9010]} & {[0.8206,0.9454]} & {[0.1380,0.9128]} & -
\end{array}\right)
$$




\section{A general decision making algorithm based on interval-valued fuzzy pref- erence relations}

Next, we present the three first steps to solve decision making problems using $\mathcal{I} \mathcal{V} \mathcal{F} \mathcal{R}$ from a given fuzzy preference relation $\mathcal{R}^{*}$ and the lack of knowledge of the expert when given the values of such relation, $g$ :

1. Using the normalisation Equation (5), we obtain from $\mathcal{R}^{*}$ the reciprocal preference relation $\mathcal{R}$;

2. The interval-valued fuzzy preference relation $\widetilde{\mathcal{R}}$ is built using $F_{T_{P}, S_{+}}\left(\mathcal{R}_{i j}, g\left(\mathcal{R}^{*}{ }_{i j}\right)\right)$;

3. The strict interval-valued fuzzy preference relation $\mathbb{P}$ associated to $\widetilde{\mathcal{R}}$ is computed using Equation (8).

The ranking of the alternatives can be done by applying a particular selection strategy to $\mathbb{P}$. In Section 9, we describe a general selection method based on the use of aggregation functions, followed by an analysis of the following particular cases: the weighted voting procedure $([21,29])$; the OWA operator with crisp weights [49]; the OWA operator with interval weights [53]; and the Non-dominance criterion $([22,36])$, among others.

Our proposal, for the first three steps, is the following:

Input: $X=\left\{x_{1}, \cdots, x_{n}\right\}$ set of alternatives; $\mathcal{R}^{*} \in \mathcal{F} \mathcal{R}(X \times X)$ : fuzzy preference relation, $g$ : weak ignorance function; $T_{a}, T_{b}$ : two t-norms in $[0,1]$ such that $T_{a} \leq T_{b}$

Output: $\mathbb{P}:$ An interval-valued strict fuzzy preference relation.

(IVFR1) Obtain $\mathcal{R} \in \mathcal{F} \mathcal{R}(X \times X)$ from $\mathcal{R}^{*} \in \mathcal{F} \mathcal{R}(X \times X)$ using Eq. (5);

(IVFR2) Build $\widetilde{\mathcal{R}} \in \mathcal{I} \mathcal{V} \mathcal{F} \mathcal{R}(X \times X)$ as follows:

$$
\mathbf{r}_{i j}=F_{T_{P}, S_{+}}\left(\mathcal{R}_{i j}, g\left(\mathcal{R}_{i j}^{*}\right)\right)
$$

(IVFR3) Construct the interval-valued strict fuzzy preference relation, $\mathbb{P}$, using Eq. (8):

$$
\mathbb{P}_{i j}=T_{I V}\left(\mathbf{r}_{i j}, N_{I V}\left(\mathbf{r}_{j i}\right)\right)=\left[T_{a}\left(\underline{r}_{i j}, 1-\bar{r}_{j i}\right), T_{b}\left(\bar{r}_{i j}, 1-\underline{r}_{j i}\right) ;\right]
$$

\section{Algorithm 1: IVFR Algorithm}

The following two examples are provided for illustration purposes:

Example 5. Assuming: $X=\left\{x_{1}, x_{2}, x_{3}, x_{4}\right\} ; \mathcal{R}^{*}$ and $g$ the fuzzy preference relation and ignorance function given in Example 4; and $T_{a}=T_{b}=T_{M}$.

(IVFR1) $\mathcal{R}=\mathcal{R}^{*}\left(\mathcal{R}^{*}\right.$ is reciprocal)

(IVFR2) $\widetilde{\mathcal{R}}=\left(\begin{array}{cccc}- & {[0.1166,0.7464]} & {[0.1090,0.4300]} & {[0.0990,0.8270]} \\ {[0.2536,0.8834]} & - & {[0.0374,0.1188]} & {[0.0546,0.1794]} \\ {[0.5700,0.8910]} & {[0.8812,0.9626]} & - & {[0.0872,0.8620]} \\ {[0.1730,0.9010]} & {[0.8206,0.9454]} & {[0.1380,0.9128]} & -\end{array}\right)$

(IVFR3) $\mathbb{P}=\widetilde{\mathcal{R}}($ Theorem 6) 
Example 6. Assume now that we have the same input than in Example 5 but for the t-norms: $T_{a}=T_{b}=T_{E}$. Note that the only difference wrt Example 5 is step (IVFR3), i.e. the construction of $\mathbb{P}$ using the Lukasiewicz t-norm:

(IVFR3) $\mathbb{P}=\left(\begin{array}{cccc}- & {[0,0.4928]} & {[0,0]} & {[0,0.654]} \\ {[0,0.7668]} & - & {[0,0]} & {[0,0]} \\ {[0.14,0.782]} & {[0.7624,0.9252]} & - & {[0,0.724]} \\ {[0,0.802]} & {[0.6412,0.8908]} & {[0,0.8256]} & -\end{array}\right)$

\section{Selection of an alternative}

Once Algorithm 1 has been executed, the following two steps selection algorithm is applied to get the final solution alternative to the decision making problem:

Input: $\mathbb{P}:$ An interval-valued strict fuzzy preference relation.

Output: Solution alternative: $x_{\text {selection }}$

(IVFR4) Select an admissible $\preceq$ order between intervals;

(IVFR5) Choose an alternative from $X$ using an interval aggregation function $\mathcal{M}$

and the admissible order $\preceq$ :

$$
x_{\text {selection }}=\underset{k}{\arg \max } \underset{\substack{k, l \in\{1, \cdots, n\} \\ 1 \leq l \neq k \leq n}}{\mathcal{M}} \mathbb{P}_{k l}
$$

\section{Algorithm 2: Selection Algorithm}

Different approaches are possible in step (IVFR5). IN the following, some of the numerical approaches mostly used in the literature to select an alternative are generalised to the case of interval values.

\subsection{Case 1: Voting method}

If we take as aggregation function the arithmetic mean we obtain a generalisation of the well known numerical voting-decision strategy to the case of interval values. This first technique is one of the simplest and most widely used aggregation method in pairwise learning [30]. The final class is assigned by computing the maximum vote by rows from the values of the strict fuzzy preference relation $\mathbb{P}$.

A generalisation of this method is presented in [29] where the authors consider a fuzzy preference structure $(\mathcal{P}, \mathcal{I}, \mathcal{J})$. Again, this method is generalised here by using the interval-valued concepts of strict preference relation $(\mathbb{P})$, indifference relation $(\mathbb{I})$ and incomparability relation $(\mathbb{J})$, yielding the following expression:

$$
x_{\text {selection }}=\underset{k}{\arg \max } \underset{\substack{k, l \in\{1, \cdots, n\} \\ 1 \leq l \neq k \leq n}}{\mathcal{M}} \sum \mathbb{P}_{k l}-\frac{1}{2} \mathbb{I}_{k l}+\frac{N_{k}}{N_{k}+N_{l}} \mathbb{J}_{k l} .
$$

Recall that the construction of both $\mathbb{I}$ and $\mathbb{J}$ use the same the t-norms $T_{a}, T_{b}$ used to construct $\mathbb{P}$ to maintain the concept of preference structure. 


\subsection{Case 2: OWA operators with numeric weights}

In 1988, Yager introduced an aggregation technique based on the ordered weighted averaging (OWA) scheme.

Definition 15 (Yager [49]). An OWA operator of dimension $n$ is a mapping $\Phi:[0,1]^{n} \rightarrow$ $[0,1]$, which has an associated set of weights $W=\left(w_{1}, \cdots w_{n}\right)^{T}$ to it, so that $w_{i} \in[0,1]$ and $\sum_{i=1}^{n} w_{i}=1$,

$$
\Phi(a)=\Phi\left(a_{1}, \cdots, a_{n}\right)=\sum_{i=1}^{n} w_{i} \cdot b_{j}
$$

where $b_{j}$ is the $j$-th largest element of the set $\left\{a_{1}, \cdots, a_{n}\right\}$.

OWA operators can incorporate the concept of fuzzy majority by means of a relative linguistic quantifier $[31,32,50,52]$ used to compute the weighting vector $W$. A nondecreasing proportional quantifier is a mapping $Q:[0,1] \rightarrow[0,1]$ with $Q(0)=0$, and such that there exists $r \in[0,1]$ for which $Q(r)=1$. Non-decreasing proportional quantifiers can be used to build membership functions as follows ([52]):

$$
Q(r)= \begin{cases}0 & \text { if } 0 \leq r<a \\ \frac{r-a}{b-a} & \text { if } a \leq r \leq b \\ 1 & \text { if } b<r \leq 1\end{cases}
$$

with $a, b, r \in[0,1]$.

Some of the most used linguistic quantifiers are "at least half", "most of" and "as many as possible", which can be represented using the values $(0,0.5),(0.3,0.8)$ and $(0.5,1)$ for $(a, b)$, respectively [14, 27, 28, 31, 32, 40].

red The linguistic quantifier that represents the concept of fuzzy majority is used to calculate the weighting vector of $\Phi, W=\left(w_{1}, \ldots, w_{n}\right)$ according to the following expression [49]:

$$
w_{i}=Q\left(\frac{i}{n}\right)-Q\left(\frac{i-1}{n}\right)
$$

Therefore, an OWA operator guided by a linguistic quantifier $Q$ representing the concept of fuzzy majority to implement in the decision making problem can be applied in step (IVFR5). Obviously, the order chosen in step (IVFR4) is used:

- in each row, to establish the order between the intervals before applying the OWA operator; and

- for producing the final ranking of the alternatives.

It is necessary to remark that if all the weights are equal we obtain the voting method with the arithmetic mean described in Subsection 9.1.

Alternative representations for the concept of fuzzy majority can be found in the literature. For example, Yager in [50] considered the family of non-decreasing proportional quantifiers $Q(r)=r^{a}(a \geq 0)$ for such representation. This family of quantifiers guarantees that [15]: (i) all the experts contribute to the final aggregated value (strict monotonicity property), and (ii) associates, when $a \in[0,1]$, higher weight values to the aggregated values with associated higher importance values (concavity property). 


\subsection{Case 3: OWA operators with interval weights}

In most of the applications of interval-valued OWA operators, the weights are taken as real numbers. However, in the presence of uncertainty the knowledge of the weights values with total precision is questionable [53, 54]. Therefore, there are cases when it might be necessary and practical to carry out aggregation of uncertain information with uncertain weights.

In the following, interval-valued weights are built from numerical weights using Proposition 2, Theorem 7 and weak ignorance functions. Given $W=\left(w_{1}, \cdots w_{n}\right)$ a weighting vector such that $w_{i} \in[0,1]$ and $\sum_{i=1}^{n} w_{i}=1$, interval weights are calculated as follows:

$$
\overline{\mathbf{w}}_{i}=F_{T_{P}, S_{+}}\left(w_{i}, g\left(w_{i}\right)\right)=\left[w_{i} \cdot\left(1-g\left(w_{i}\right)\right), w_{i}+g\left(w_{i}\right)-w_{i} \cdot g\left(w_{i}\right)\right] .
$$

To comply with the addition of 1 of the numerical weights, the sum of the upper bounds of the interval-valued weights is also set to be equal to 1 . To achieve this constrain, the interval-valued weights are normalised as follows:

$$
\overline{\mathbf{w}}_{i}=\left[\frac{w_{i} \cdot\left(1-g\left(w_{i}\right)\right)}{S_{+_{T}}}, \frac{w_{i}+g\left(w_{i}\right)-w_{i} \cdot g\left(w_{i}\right)}{S_{+_{T}}}\right],
$$

with

$$
S_{+_{T}}=\sum_{i=1}^{n} S_{+}\left(w_{i}, g\left(w_{i}\right)\right)=\sum_{i=1}^{n}\left[w_{i}+g\left(w_{i}\right)-w_{i} \cdot g\left(w_{i}\right)\right]=1+\sum_{i=1}^{n}\left(1-w_{i}\right) \cdot g\left(w_{i}\right) .
$$

In the particular case of using the weak ignorance function $g(x)=2 \cdot \min (x, 1-x)$, we observe the following:

1. If $w_{1}=\cdots=w_{n}=\frac{1}{n}(n \geq 2)$, then $\overline{\mathbf{w}}_{\mathbf{i}}=\frac{1}{n} \cdot\left[\frac{n-2}{3 \cdot n-2}, 1\right] \forall i$.

2. If $w_{1}=1$ and $w_{j}=0 \forall j>1(n \geq 2)$, then $\overline{\mathbf{w}}_{\mathbf{1}}=[1,1]$, and $\overline{\mathbf{w}}_{\mathbf{j}}=[0,0] \forall j>1$.

Note that if a linguistic quantifier $Q$ is used to compute the numerical weights $w_{i}$ then Eq. (19) can be rewritten in terms of $Q$ and $g$ using Eq. (18). These will be referred to as the quantifier guided interval-valued weights. After the normalisation process described above is being applied we get the corresponding normalised quantifier guided intervalvalued weights.

\subsection{Case 4: Non-dominance criterion}

In this subsection we present a generalisation of the non-dominance criterion given by

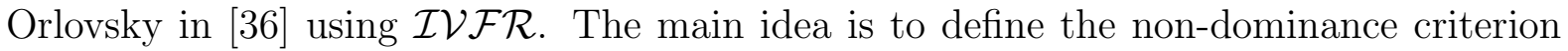
using interval-valued fuzzy preference relations to derive an ordering of the alternatives and therefore to allow the selection of the 'best' alternatives. We must point out that it could happen that there exist two or more alternatives with the same non-dominance degree, i.e. a total ordering of the set of alternatives is not guaranteed. This fact has led many authors to propose alternative algorithms (see [22, 27, 43, 48]).

Next, we propose an alternative selection algorithm that replaces step (IVFR5) of Algorithm 2 using the non-dominance concept applied to $\mathcal{I V \mathcal { F } R}$. The new selection algorithm is given below: 
(ND-IVFR5) Build the non-dominance interval-valued fuzzy set:

$$
\begin{aligned}
& N D_{I V}=\left\{\left(x_{j}, N D_{I V}\left(x_{j}\right)\right) \mid x_{j} \in X\right\} \\
& N D_{I V}\left(x_{j}\right)=\left[\bigvee_{i=1}^{n}\left(\underline{r}_{i j}\right), \bigvee_{i=1}^{n}\left(\bar{r}_{i j}\right)\right]
\end{aligned}
$$

(ND-IVFR6) Apply $N_{I V}$, generated by the standard strong negation, to the set $N D_{I V}$ :

$$
N_{I V}\left(N D_{I V}\right)\left(x_{j}\right)=\left[1-\bigvee_{i=1}^{n}\left(\bar{r}_{i j}\right), 1-\bigvee_{i=1}^{n}\left(\underline{r}_{i j}\right)\right]
$$

(ND-IVFR7) Order the elements of the set $N_{I V}\left(N D_{I V}\right)$ in a decreasing way with respect to the admissible order of the membership intervals selected in (IVFR4);

Algorithm 3: Non-dominance Selection Algorithm

\section{Consensus}

Next we present an algorithm for those cases in which, after using different methods to choose an alternative, we have more than one alternative tied as 'best'. Thus the following algorithm is used to brake the and select the final solution of the decision making problem.

(C1) if most of the methods return the same alternative $x_{i}$ as 'best' solution then

choose $x_{i}$ as the final solution of the decision making problem; else

(C2) Choose among the tied alternatives the one with smallest interval length as the final solution of the decision making problem;

end

\section{Algorithm 4: Consensus Algorithm}

The rationale of (C2) resides in the interpretation of the the length of the interval as a measure of the lack of knowledge of the expert when providing the membership degree of an element to a fuzzy set $[10,11,42]$.

\section{Illustrative example}

Following Example 5 and the $\mathcal{I} \mathcal{V P} \mathcal{R}, \widetilde{\mathcal{R}}$, obtained there, we present the results the application of the Selection Algorithm 2 results in at each one of the four cases described in Section 9, when the following admissible orders between intervals are used: $\preceq_{L 1}, \preceq_{L 2}$ ,$\preceq_{X Y}$. Obviously, in the case of the non-dominance criterion the selection will be made applying tyne corresponding Algorithm 3. Finally, in case of having a tie between two or more alternatives the Consensus Algorithm 4 will be applied.

\subsection{Voting method}

Table 1 show the sum of the interval values of each row of $\widetilde{\mathcal{R}}$. 


\begin{tabular}{|c|c|c|c|}
\hline$x_{1}$ & $x_{2}$ & $x_{3}$ & $x_{4}$ \\
\hline \hline$[0.1082,0.6678]$ & {$[0.1152,0.3939]$} & {$[0.5128,0.9052]$} & {$[0.3772,0.9197]$} \\
\hline
\end{tabular}

Table 1: Voting method: The interval aggregation function for each row of $\widetilde{\mathcal{R}}$

Table 2 provides the ordering of the alternatives for each one of the three admissible orders between intervals, $\preceq_{L 1}, \preceq_{L 2}, \preceq_{X Y}$, with the interval aggregation operator $\mathcal{M}$ being being the arithmetic mean.

\begin{tabular}{|c|c|c|}
\hline$\preceq_{L 1}$ & $\preceq_{L 2}$ & $\preceq_{X Y}$ \\
\hline \hline$x_{1} \preceq_{L 1} x_{2} \preceq_{L 1} x_{4} \preceq_{L 1} x_{3}$ & $x_{2} \preceq_{L 2} x_{1} \preceq_{L 2} x_{3} \preceq_{L 2} x_{4}$ & $x_{2} \preceq_{X Y} x_{1} \preceq_{X Y} x_{4} \preceq_{X Y} x_{3}$ \\
\hline
\end{tabular}

Table 2: Voting method: Ordering of alternatives for the three different admissible orders

\subsection{OWA operator with numeric weights}

The results obtained by applying Selection Algorithm 2 to $\widetilde{\mathcal{R}}$ using the OWA operator "at least half", and the admissible orders between intervals $\preceq_{L 1}, \preceq_{L 2}, \preceq_{X Y}$ is given in Table 3. For information, the numeric weights associated to this OWA operator are $w_{1}=0.7, w_{2}=0.3, w_{3}=0$.

\begin{tabular}{|c|c|c|c|}
\hline & $\preceq_{L 1}$ & $\preceq_{L 2}$ & $\preceq_{X Y}$ \\
\hline \hline$x_{1}$ & {$[0.1143,0.6515]$} & {$[0.1043,0.8028]$} & {$[0.1043,0.8028]$} \\
\hline$x_{2}$ & {$[0.1939,0.6722]$} & {$[0.1939,0.6722]$} & {$[0.0494,0.1612]$} \\
\hline$x_{3}$ & {$[0.7878,0.9411]$} & {$[0.7878,0.9411]$} & {$[0.7878,0.9411]$} \\
\hline$x_{4}$ & {$[0.6263,0.9321]$} & {$[0.6158,0.9356]$} & {$[0.6263,0.9321]$} \\
\hline
\end{tabular}

Table 3: OWA operator "at least half": Results for $\widetilde{\mathcal{R}}$ and admissible orders $\preceq_{L 1}, \preceq_{L 2}$ and $\preceq_{X Y}$.

Table 4 provides the ordering of the alternatives for each one of the three admissible orders between intervals, $\preceq_{L 1}, \preceq_{L 2}, \preceq_{X Y}$, applied to the data of Table 3 .

\subsection{OWA operators with interval weights}

Taking $g(x)=2 \cdot \min (x, 1-x)$ as the weak ignorance function and the same OWA operator "at least half" with numeric weights as above $w_{1}=0.7, w_{2}=0.3$, and $w_{3}=0$, we have the following quantifier guided interval-valued weights

$$
\bar{w}_{1}=[0.28,0.88], \bar{w}_{2}=[0.12,0.72], \bar{w}_{3}=[0,0] .
$$

. Because $S_{+_{T}}=1.6$, the normalised quantifier guided interval weights are :

$$
\overline{\mathbf{w}}_{\mathbf{1}}=[0.175,0.55], \overline{\mathbf{w}}_{\mathbf{2}}=[0.075,0.45], \overline{\mathbf{w}}_{\mathbf{3}}=[0,0] .
$$

The results obtained by applying Selection Algorithm 2 to $\widetilde{\mathcal{R}}$ using the OWA operator "at least half" with these interval weights, and the admissible orders between intervals $\preceq_{L 1}, \preceq_{L 2}, \preceq_{X Y}$ is given in Table 5 .

Table 6 provides the ordering of the alternatives for each one of the three admissible orders between intervals, $\preceq_{L 1}, \preceq_{L 2}, \preceq_{X Y}$, applied to the data of Table 5 . 


\begin{tabular}{|c|c|c|}
\hline$\preceq_{L 1}$ & $\preceq_{L 2}$ & $\preceq_{X Y}$ \\
\hline \hline$x_{1} \preceq_{L 1} x_{2} \preceq_{L 1} x_{4} \preceq_{L 1} x_{3}$ & $x_{2} \preceq_{L 2} x_{1} \preceq_{L 2} x_{4} \preceq_{L 2} x_{3}$ & $x_{2} \preceq_{X Y} x_{1} \preceq_{X Y} x_{4} \preceq_{X Y} x_{3}$ \\
\hline
\end{tabular}

Table 4: OWA operator "at least half": Ordering of alternatives for the three different admissible orders

\begin{tabular}{|c|c|c|c|}
\hline & $\preceq_{L 1}$ & $\preceq_{L 2}$ & $\preceq_{X Y}$ \\
\hline \hline$x_{1}$ & {$[0.0286,0.6040]$} & {$[0.0261,0.7907]$} & {$[0.0261,0.7907]$} \\
\hline$x_{2}$ & {$[0.0485,0.5666]$} & {$[0.0485,0.5666]$} & {$[0.0485,0.5666]$} \\
\hline$x_{3}$ & {$[0.1970,0.9304]$} & {$[0.1970,0.9304]$} & {$[0.1970,0.9304]$} \\
\hline$x_{4}$ & {$[0.1566,0.9254]$} & {$[0.1540,0.9307]$} & {$[0.1566,0.9254]$} \\
\hline
\end{tabular}

Table 5: OWA operator "at least half" with interval weights: Results for $\widetilde{\mathcal{R}}$ and admissible orders $\preceq_{L 1}$, $\preceq_{L 2}$ and $\preceq_{X Y}$.

\subsection{Non-dominance criterion}

The application of the Selection Algorithm 3 to $\widetilde{\mathcal{R}}$ with admissible orders between intervals $\preceq_{L 1}, \preceq_{L 2}, \preceq_{X Y}$ is shown below:

(ND-IVFR5) Non-dominance interval-valued fuzzy set:

$$
\begin{aligned}
& N D_{I V}=\left\{\left(x_{1},[0.5700,0.9010]\right),\left(x_{2},[0.8812,0.9626]\right),\right. \\
&\left.\left(x_{3},[0.1380,0.9128]\right),\left(x_{4},[0.0990,0.8620]\right)\right\}
\end{aligned}
$$

(ND-IVFR6) Apply $N_{I V}$ to the set $N D_{I V}$ :

$$
\begin{aligned}
N_{I V}\left(N D_{I V}\right)=\{( & \left(x_{1},[0.0990,0.4300]\right),\left(x_{2},[0.0374,0.1188]\right), \\
& \left.\left(x_{3},[0.0872,0.8620]\right),\left(x_{4},[0.1380,0.9010]\right)\right\}
\end{aligned}
$$

(ND-IVFR7) Ordering of alternatives:

Finally, we observe that in seven cases we obtain $x_{3}$ as the 'best' solution alternative, while $x_{4}$ is classed as 'best' in five cases the alternative. The application of the first rule $(C 1)$ of the Consensus Algorithm 4 leads to the selection alternative $x_{3}$ as the final solution of the decision making problem. If both alternatives had been declared 'best' in the same number of cases (six each) then we would have to apply the second rule (C2) of the of the Consensus Algorithm 4 to brake the tie, an option that is not possible in the numerical case.

\section{Conclusions}

In this paper we have presented different approaches to solve decision making problems based on the use of interval-valued fuzzy preference relations. Taking a fuzzy preference relation as a starting point and (1) using weak ignorance functions to penalise indifference situations; that is, situations in which the preference of one alternative against another is close to 0.5 ; and (2) representing the preference degree of a relation by means of interval values with length equal to the weak ignorance of the expert when he or she assigns a numeric preference value, we have developed and designed decision making procedures to select the 'best' alternative solution to the decision making problem. 


\begin{tabular}{|c|c|c|}
\hline$\preceq_{L 1}$ & $\preceq_{L 2}$ & $\preceq_{X Y}$ \\
\hline$x_{1} \preceq_{L 1} x_{2} \preceq_{L 1} x_{4} \preceq_{L 1} x_{3}$ & $x_{2} \preceq_{L 2} x_{1} \preceq_{L 2} x_{3} \preceq_{L 2} x_{4}$ & $x_{2} \preceq_{X Y} x_{1} \preceq_{X Y} x_{4} \preceq_{X Y} x_{3}$ \\
\hline
\end{tabular}

Table 6: OWA operator "at least half" with interval weights: Ordering of alternatives for the three different admissible orders

\begin{tabular}{|c|c|c|}
\hline$\preceq_{L 1}$ & $\preceq_{L 2}$ & $\preceq_{X Y}$ \\
\hline \hline$x_{2} \preceq_{L 1} x_{3} \preceq_{L 1} x_{1} \preceq_{L 1} x_{4}$ & $x_{2} \preceq_{L 2} x_{1} \preceq_{L 2} x_{3} \preceq_{L 2} x_{4}$ & $x_{2} \preceq_{X Y} x_{1} \preceq_{X Y} x_{3} \preceq_{X Y} x_{4}$ \\
\hline
\end{tabular}

Table 7: Non-dominance criterion: Ordering of alternatives for the three different admissible orders

The general algorithm presented in this paper is based on a thorough study and formal design of a novel construction method of interval-valued fuzzy sets from ordinary fuzzy sets. We have proved that the product t-norm and the probabilistic sum t-conorm are the only ones that allow the generation of interval values of length equal to the weak ignorance associated with the membership degree of the same element of the original fuzzy set. This main result is the driver of a new general algorithm to build IVFR where the lack of knowledge of the expert in giving numerical preference values in the pairwise comparison of alternatives plays a crucial role. This general algorithm is complemented with different selection processes of the alternative solution of the decision making problem. Finally, we propose to construct interval weights for OWA operators considering also the weak ignorance function. Because selection processes based on interval-valued information are very much dependent of the particular admissible ordering applied, we have argued that a set of admissible orders should always be applied in the selection of the final solution of the decision making problem. This methodology could lead to the appearance of ties between different alternatives at the selection process. To overcome this issue, a consensus algorithm to break ties is proposed, an option that is not possible in the context of numerical preferences.

\section{Acknowledgment}

This work has been partially supported by the Research Services of the Universidad Publica de Navarra, and the National Science Foundation of Spain, reference TIN201015055.

[1] J. Aczél, Sur les opérations définies pour des nombres réels, Bull Soc. Math. France 76 (1949) 59-64.

[2] K. Atanassov, Intuitionistic fuzzy sets, VIIth ITKR Session, Deposited in the Central Science and Technology Library of the Bulgarian Academy of Sciences, Sofia, Bulgaria, (1983) 1684-1697.

[3] K. Atanassov, Intuitionistic fuzzy sets, Fuzzy Sets and Systems 20 (1986) 87-96.

[4] G. Beliakov, A. Pradera, T. Calvo, What is an aggregation function in Aggregation Functions: A Guide for Practitioners (Studies In Fuzziness and Soft Computing) Springer 221 (2007) 1-37.

[5] H. Bustince, P. Burillo, Structures on intuitionistic fuzzy relations, Fuzzy Sets and Systems 78 (1996) 293-303. 
[6] H. Bustince, Construction of intuitionistic fuzzy relations with predetermined properties, Fuzzy Sets and Systems 109 (2000) 379-403 .

[7] H. Bustince, P. Burillo, Mathematical analysis of interval-valued fuzzy relations: Application to approximate reasoning, Fuzzy Sets and Systems 113 (2000) 205-219.

[8] H. Bustince, E. Barrenechea, M. Pagola, Relationship between restricted dissimilarity functions, restricted equivalence functions and normal $E_{N}$-functions: Image thresholding invariant, Pattern Recognition Letters 29 (2008) 525-536.

[9] H. Bustince, E. Barrenechea, M. Pagola, Generation of interval-valued fuzzy and Atanassov's intuitionistic fuzzy connectives from fuzzy connectives and from $K_{\alpha}$ operators: laws for conjunctions and disjunctions, amplitude, International Journal of Intelligent Systems 23 (6) (2008) 680-714.

[10] H. Bustince, E. Barrenechea, M. Pagola, J. Fernandez, Interval-valued fuzzy sets constructed from matrices: application to edge detection, Fuzzy Sets and Systems 160 (13) (2009) 1819-1840.

[11] H. Bustince, M. Pagola, E. Barrenechea, J. Fernandez, P. Melo-Pinto, P. Couto, H.R. Tizhoosh, J. Montero, Ignorance functions. An application to the calculation of the threshold in prostate ultrasound images, Fuzzy Sets and Systems 161 (1) (2010) $20-36$.

[12] H. Bustince, J. Fernandez, A. Kolesárová, R. Mesiar, Generation of linear orders for intervals by means of aggregation functions, Fuzzy Sets and Systems (2012) http://dx.doi.org/10.1016/j.fss.2012.07.015

[13] T. Calvo, A. Kolesárová, M. Komornikova, R. Mesiar, Aggregation operators: properties, classes and construction methods in Aggregation Operators New Trends and Applications Physica-Verlag Heidelberg (2002) 3-104.

[14] F. Chiclana, F. Herrera, and E. Herrera-Viedma, Integrating three representation models in fuzzy multipurpose decision making based on fuzzy preference relations, Fuzzy Sets and Systems 97(1) (1998) 33-48.

[15] F. Chiclana, E. Herrera-Viedma, F. Herrera, and S. Alonso, Some induced ordered weighted averaging operators and their use for solving group decision-making problems based on fuzzy preference relations, European Journal of Operational Research 182(1) (2007) 383-399.

[16] B. De Baets, B. Van de Walle, E. Kerre, Fuzzy preference structures without incomparability, Fuzzy Sets and Systems 76 (3) (1995) 333-348.

[17] B. De Baets, B. Van de Walle, E. Kerre, Fuzzy preference structures and their characterization, Journal of Fuzzy Mathematics 3 (1995) 373-381.

[18] G. Deschrijver, E.E. Kerre, On the composition of intuitionistic fuzzy relations, Fuzzy Sets and Systems 136 (2003) 333-361.

[19] G. Deschrijver, C. Cornelis, E.E. Kerre, On the representation of intuitionistic fuzzy T-norms and T-conorms, IEEE Transactions on Fuzzy Systems 12(1) (2004) 45-61. 
[20] D. Dubois, W. Ostasiewicz, H. Prade, Fuzzy Sets: History and Basic Notions in Fundamentals of Fuzzy Sets, Kluwer, Boston, MA (2000) 21-124.

[21] M. Dummett, A Voting Procedures, Oxford, U.K., Clarendon (1984).

[22] A. Fernández, M. Calderón, E. Barrenechea, H. Bustince, F. Herrera, Solving multiclass problems with linguistic fuzzy rule based classification systems based on pairwise learning and preference relations, Fuzzy Sets and Systems 161(23) (2010) 3064-3080.

[23] J. Fodor, M. Roubens, Fuzzy Preference Modelling and Multicriteria Decision Support in Theory and Decision Library, Kluwer Academic Publishers (1994).

[24] J. Fodor, M. Roubens, Valued preference structures, European Journal of Operational Research 79 (1994) 277-286.

[25] M.B. Gorzalczany, A method of inference in approximate reasoning based on intervalvalued fuzzy sets, Fuzzy Sets and Systems 21 (1987) 1-17.

[26] M. Hanss, Applied Fuzzy Arithmetic: An Introduc tion with Engineering Applications. By. Michael Hanss. Springer-Verlag, Berlin (2005).

[27] F. Herrera, L. Martínez, P. J. Sánchez, Managing non-homogeneous information in group decision making, European Journal of Operational Research 166 (1) (2005) $115-132$.

[28] F. Herrera, S. Alonso, F. Chiclana, E. Herrera-Viedma E, Computing with words in decision making: foundations, trends and prospects, Fuzzy Optimization and Decision Making 8 (2009) 337-364.

[29] E. Hüllermeier, K. Brinker, Learning valued preference structures for solving classification problems, Fuzzy Sets and Systems 159 (18) (2008) 2337-2352.

[30] E. Hüllermeier, S. Vanderlooy, Combining predictions in pairwise classification: an optimal adaptive voting strategy and its relation to weighted voting, Pattern Recognition 43(1) (2010) 128-142.

[31] J. Kacprzyk, Group decision making with a fuzzy linguistic majority, Fuzzy Sets and Systems 18 (1986) 105-118.

[32] J. Kacprzyk, M. Fedrizzi, H. Nurmi, OWA operators in group decision making and consensus reaching under fuzzy preferences and fuzzy majority in The Ordered Weighted Averaging Operators: Theory and Applications, Kluwer Academic Publishers, Dordrecht, (1997) 193-206.

[33] E.P. Klement, R. Mesiar, E. Pap, Triangular norms, Kluwer, Dordretch, (2000).

[34] J.M. Mendel, R.I.J. Robert, Type-2 fuzzy sets made simple, IEEE Transactions on Fuzzy Systems 10 (2002) 117-127.

[35] J. Montero, D. Gómez, H. Bustince, On the relevance of some families of fuzzy sets, Fuzzy Sets and Systems 158 (2007) 2429-2442.

[36] S. A. Orlovsky, Decision-making with a fuzzy preference relation, Fuzzy Sets and Systems 1 (3) (1978) 155-167. 
[37] S. V. Ovchinnikov, M. Roubens, On Strict Preference Relations, Fuzzy Sets and Systems 43 (1991) 319-326.

[38] S. V. Ovchinnikov, V. M. Ozernoy, Using fuzzy binary relations for identifying noninferior decision alternatives, Fuzzy Sets and Systems 25 (1988) 21-32.

[39] P. Perny, B. Roy, The use of fuzzy outranking relations in preference modelling, Fuzzy Sets and Systems 49 (1992) 33-53.

[40] G. Sanchez-Hernandez, F. Chiclana, N. Agell, J. C. Aguado, Ranking and selection of unsupervised learning marketing segmentation, Knowledge-Based Systems 44 (2013) 20-33.

[41] J. Sanz, A. Fernández, H. Bustince, F. Herrera, A genetic tuning to improve the performance of fuzzy rule-based classification systems with interval-valued fuzzy sets: Degree of ignorance and lateral position, International Journal of Approximate Reasoning 52 (6) (2011) 751-766.

[42] P. Sevastianov, Numerical methods for interval and fuzzy number comparison based on the probabilistic approach and Dempster-Shafer theory, Information Sciences 177 (2007) 4645-4661.

[43] E. Szmidt, J. Kacprzyk, Using intuitionistic fuzzy sets in group decision making, Control and Cybernetics 78 (1996) 183-195.

[44] I.B. Türksen, T. Bilgiç, Interval-valued strict preference relations with Zadeh triples, Fuzzy Sets and Systems 78 (1996) 183-195.

[45] I.B. Türksen, Type 2 representation and reasoning for computing with words, Fuzzy Sets and Systems 127 (2002) 17-36.

[46] Z.S. Xu, On compatibility of interval fuzzy preference relations, Fuzzy Optimization and Decision Making 3 (2004) 217-225.

[47] Z.S. Xu, R.R. Yager, Some geometric aggregation operators based on intuitionistic fuzzy sets, International Journal of General Systems 35 (2006) 417-433.

[48] Z.S. Xu, A method based on distance measure for interval-valued intuitionistic fuzzy group decision making, Information Sciences 180 (1) (2010) 181-190.

[49] R.R. Yager, On ordered weighted averaging aggregation operators in multicriteria decision making, IEEE Transactions on Systems, Man, and Cybernetics 18 (1988) $183-190$

[50] R.R. Yager, Quantifier guided aggregation using OWA operators, International Journal of Intelligent Systems 11 (1996) 49-73.

[51] L.A. Zadeh, The concept of a linguistic variable and its application to approximate reasoning I, Information Sciences 8 (1975) 199-249.

[52] L.A. Zadeh, A computational approach to fuzzy quantifiers in natural languages, Computers \& Mathematics with Applications 9 (1983) 149-184. 
[53] S.-M. Zhou, F. Chiclana, R. I. John, J. M. Garibaldi, Type-1 OWA operators for aggregating uncertain information with uncertain weights induced by type-2 linguistic quantifiers, Fuzzy Sets and Systems 159 (2008) 3281-3296.

[54] S. -M. Zhou, F. Chiclana, R. I. John and J. M. Garibaldi, Alpha-Level Aggregation: A Practical Approach to Type-1 OWA Operation for Aggregating Uncertain Information with Applications to Breast Cancer Treatments, IEEE Transactions on Knowledge and Data Engineering 23 (10) (2011)1455-1468. 